\title{
INSULIN THERAPY IN SEPSIS
}

\author{
Vilian Platikanov, Nikoleta Slavova, Maria Todorova \\ Department of Anesthesiology and Intensive Care, \\ St. Marina University Hospital of Varna
}

\begin{abstract}
Severe metabolic derangement is characteristic of systemic inflammatory response in septic conditions. Changes in blood glucose levels are often deleterious further impairing organ functioning. Thus they are a subject of strict and sometimes even too aggressive control. Its disadvantages demand and stimulate research of new strategies. In the present paper we systematically review the current therapeutic options and recent research advances in the field of hyperglycemia in sepsis. They include the pathogenesis of sepsis and the role of insulin in this pathology. A special attention is paid to the glycemic control in sepsis. The correct strategy of glycemic control is combined approach with computerized infusion, continuous blood glucose monitoring and proper feeding support.
\end{abstract}

Key words: sepsis, intensive care, insulin therapy, glycemic control, nutritional support

\section{INTRODUCTION}

Providing full medical support enhancing the recovery is crucial for patients suffering from sepsis. Optimal intensive care requires correction of disbalance produced by the septic condition. Elevated blood glucose even in patients without diabetes mellitus is one of the most frequent findings with numerous adverse effects.

\section{PATHOGENESIS OF SEPSIS}

In septic patients pathogens' invasion threatens homeostasis. As a sophisticated and self-organized unit the human body responses to the threat by means of an adaptive mechanism first described by Hans Selye in 1936. First, vital organs such as brain, heart, and kidneys are energy-supplied with great priority. This secondarily increases the options for handling

\footnotetext{
Address for correspondence:

Nikoleta Slavova, MD

Department of Anesthesiology and Intensive Care,

St. Marina University Hospital of Varna,

1 Hristo Smirnenski Str., Varna 9010, Bulgaria

E-mail:mi6cata@gmail.com
}

Received: August 30, 2012

Accepted: January 15, 2013 the invasion. The ultimate goal is restoration of the metabolic balance. Deeper insight into this response reveals a complicated cascade of neuroendocrine and proinflammatory changes. This results in an increase of circulating cytokines as interleukin (IL)1, IL-6, tumour necrosis factor (TNF)- $\alpha$, macrophage migration inhibitory factor (MIF), free radicals, nitric oxide $(\mathrm{NO})$ having deleterious effects on coagulation cascade, cardiovascular system, kidneys, lungs, liver, central nervous system, and gastrointestinal tract and leads to multiorgan dysfunction.

The pathophysiologic mechanism causes changes in carbohydrate metabolism such as insulin resistance, induction of glycogenolysis in the liver and increase of glyconeogenesis. This is the reason why even non-diabetes mellitus patients with sepsis develop hyperglycemia.

The inflammatory mediators may overcome the normal physiology of down regulation of GLUTtransporters in hyperglycemia, which exposes cell to high uptake of glucose and glucose toxicity, respectivelly. This causes hyperglycemia-induced mitochondrial abnormalities observed in liver and skeletal muscle. Superoxide production is increased in hyperglycemia. That leads to nitration of majority 
of the mitochondrial complexes and voltagedependent anion channels. High blood glucose (BG) impairs neutrophil chemotaxis and phagocytosis and decreases microvascular responsiveness $(4,8)$.

\section{INSULIN IN SEPSIS}

Beneficial effects of insulin therapy in sepsis may originate from achieving normal BG levels or the insulin itself, or both. Detrimental hyperglycemia effects are reduced or avoided in euglycemia. On the other hand, insulin acts as a powerful anabolic and antiinflammatory agent suppressing the production of IL-1, IL-6, MIF, reversing endothelial dysfunction and stimulating production of antiinflammatory factors IL-4 and IL-10.

\section{Goals of glycemic control}

Normal BG levels are strongly recommended in critically ill and particularly septic patients. It is welldocumented that hyperglycemia in these subjects is associated with higher infection rates, higher overall morbidity and mortality. A large study proved that each $1 \mathrm{mmol} / \mathrm{L}$ increase in BG level is associated with 6-10\% increased relative risk of pneumonia, urinary tract or skin infections. In 2007, Van den Berghe suggested that a range between $4,4 \mathrm{mmol} / \mathrm{L}$ and 6,1 $\mathrm{mmol} / \mathrm{L}$ should be targeted for intensive care patients (18). According to the 2008 international guidelines, target BG level should be below $8,3 \mathrm{mmol} / \mathrm{L}$ (5).

Normoglycemia is not the only goal regarding glycemic control. Of great importance is also maintaining stable BG concentration. Variations of glucose levels are a significant and independent predictor of mortality and even stronger predictor of intensive care unit (ICU) mortality than mean BG concentration $(1,6,17)$.

\section{Coagulation cascade}

Activating the endothelium through proinflammatory mediators unleashes a procoagulant state that can lead to disseminated intravascular coagulation. Hyperglycemia itself enhances coagulation and attenuates fibrinolytic activity.

\section{Myocardial function}

Myocardial depression is frequent in patients with sepsis. Moreover, this dysfunction is not related to BG concentrations and is completely reversible by intraarterial insulin infusions despite various BG concentrations. This leads to the conclusion that it is insulin that improves cardiac performance irrespective of BG levels (4).

\section{Lipid metabolism}

Critical disease is associated with lipid disorders, including increase of triglycerides and reduction of high-density lipoproteins. Insulin reverses this disbalance. Elevated free fatty acids are also proved to decline during insulin therapy (3).

\section{Renal function}

Sepsis is responsible for acute kidney injury in several mechanisms including afferent arteriolar vasoconstriction and/or efferent arteriolar vasodilatation, endotoxin and inflammatory mediators' injury, and glomerular microthrombi. Data show that intensive insulin therapy for hyperglycemic critically ill patients may help in preventing the kidney injury. Anyway, the so important fluid resuscitation should be performed with caution because of the possible deleterious effect of colloids on already impaired renal function (2).

\section{Liver function}

The reduced need of blood product transfusions may improve erythropoiesis or reduce hemolysis, since this benefit is associated with a lower incidence of hyperbilirubinemia. Intensive insulin therapy may reduce the risk of cholestasis, since adequate provision of glucose and insulin to hepatocytes is crucial for normal choleresis.

\section{Intestinal function}

Animal studies prove that insulin reduces the microcirculatory dysfunction in sepsis through lowering the platelet activating factor (PAF), PGI-2 and, especially, TxA2.

\section{GLYCEMIC CONTROL IN SEPSIS}

\section{Alternatives}

Two main alternatives of insulin therapy in ICU settings are intensive insulin treatment (IIT) and conventional insulin therapy (CIT). Significant controversies around the world regarding glycemic control originate from the very definition of IIT. The great number of publications consider both methods as intravenous insulin infusion with different target BG levels $(4,5-6,0 \mathrm{mmol} / \mathrm{L}$ in IIT and $<10 \mathrm{mmol} / \mathrm{L}$ 
in CIT) (7). In contrast, some substantial documents as the recently published Clinical Practice Guideline From the American College of Physicians define IIT as 'use of intravenous insulin to achieve targeted blood glucose level with frequent blood glucose testing and adjustment of insulin doses' with targets of 4,4-6,1 $\mathrm{mmol} / \mathrm{L}$ for ICU and $<11,1 \mathrm{mmol} / \mathrm{L}$ for non-ICU patients (14) without defining CIT.

\section{The era before Leuven}

Before 2001, no confirmation existed about the actual benefits of IIT in critically ill patients. They were subjected either to IIT, or to CIT according to institutional protocols or individual judgement.

\section{Van den Berghe's Study}

In 2001, Van den Berghe published his Leuven study of IIT in critically ill patients (19). His results showed reduced ICU and overall hospital mortality, more substantial for septic patients, lower rates of bloodstream infections, renal failure, blood product transfusions and shorter mechanical ventilation times. Because of these favourable conclusions this study became an international cornerstone for glycemic control in ICU. With time, some of these IIT advantages were proved and other emerged including reduction of fibrinolytic impairment (15), hepatoprotective effect after hepatic surgery, improved insulin sensitivity (11) and even economic benefit.

Unfortunatelly, subsequent large trials failed to confirm most of Van den Berghe's results. In 2008 and 2009, two large trials were suspended because of higher rates of adverse effects including hypoglycemia and mortality (13). Another large study (7) showed similar results. This led to still ongoing debates regarding the most appropriate glucose control method in this patients' population.

The reasons for these discrepancies may be found in the design of Van den Berghe's study. Most patients were after coronary bypass surgery. They were given intravenous loads of glucose (200 to 300 $\mathrm{g} / 24$ hours) and it was possible that IIT decreased this glucose load's adverse effects and, vise versa, this glucose load lowered the number of hypoglycemic events (7). What is more,Van den Berghe used total parenteral nutrition with his patients which also caused hyperglycemia and may have raised the mortality rate in the control arm.

One of the main concerns regarding IIT is the higher hypoglycemia rates, especially during the first 24 hours. Reported rates are different and depend on the actual protocol used and blood sample site (arterial versus capillary or venous). They are, however, always significant in IIT and independent prognostic factor for adverse effects or death. A severe hypoglycemic event ( $\mathrm{BG}<2,2 \mathrm{mmol} / \mathrm{L}$ ) means a six-fold increase in mortality (9). The number of hypoglicemias is higher in patients receiving intravenous insulin than in those receiving subcutaneous insulin. Duration of time spent in ICU is a strong predisposing factor for hypoglycemia in surgical critically ill patients. Other factors include prolonged renal replacement therapy and inotropic and vasopressor therapy (20).

The severity of sepsis is also a crucial factor regarding the rates of hypoglycemic events. Waeschle et al. find frequencies of $2,1 \%, 6 \%$ and $11,5 \%$ in non-severe sepsis, severe sepsis and septic shock, respectively (20).

This motivated the Surviving Sepsis Campaign Guidelines (5) and its Additional Statement on glucose control to recommend insulin therapy for BG over $10 \mathrm{mmol} / \mathrm{L}$ with target levels of $<8,3 \mathrm{mmol} / \mathrm{L}$ as mentioned above. Although widely debated these documents remain the current specific sepsis treatment guidelines.

Of course, success or failure of glycemic control is always somewhat influenced by the comedication in the individual patient. Certain drugs such as ACE-inhibitors or chlorpromazine can cause drop of BG levels and, eventually, hypoglycemia. Other drugs such as adrenalin, noradrenalin, $\beta$-agonists, corticosteroids, diuretics, theophylline, isoniazid, and phenytoin cause hyperglycemia. Administration regimen also influences the glycemia. A prospective randomized trial of Loisa et al. concluded that if the recommended corticosteroid therapy for septic shock is given by continuous infusion then normoglycemia is more easily achieved.

Even if IIT is the method of choice there is no uniformity in the actual protocols for glycemic control. Various more or less widely used protocols exist and each of them has its advantages and

Scripta Scientifica Medica, 2013, vol. 45 (1), pp. 7-11

Copyright (c) Medical University - Varna 
disadvantages. Differences include insulin delivery and adjustments, time to target BG levels and strategy regarding individual insulin resistance. Comparing several protocols and stating that each patient would benefit differently from any of them it was concluded that a single institutional protocol would be an advantage in terms of uniformity but it was not realistic.

\section{RECENT ADVANCES AND ADDITIONAL OPTIONS}

The majority of algorithms used for adjustment of insulin delivery include frequent blood sampling (every 1 to 4 hours) and bedside calculation of the insulin dosage. An attractive alternative may be the computerized software systems like Glucommander. Still a recent study on Glucommander use in critically ill patients showed more than twofold increase in hypoglycemia rates.

Glycemic control could be further improved by implementation of continuous or near-continuous monitoring of BG levels using intravascular or subcutaneous (tissue) glucose sensors. Interestingly, Lorencio et al. recently found out a better accuracy of real-time continuous glucose monitoring in patients with septic shock (12).

Another option is the continuous subcutaneous insulin infusion (CSII) - a successful insulin-pump method for type 1 diabetes mellitus patients.

Outcomes of IIT also depend on the nutritional support chosen. It was recently proved that permissive underfeeding (60-70\% of calculated daily caloric needs) was beneficial compared to standard feeding protocols as it lowered the mortality rates and mechanical ventilation duration.

It is possible that one of the keys towards safer IIT with lower rates of hypoglycemia is the route of feeding administration. Current clinical nutrition guidelines recommend enteral nutrition (EN) for all the patients who are not expected to be on a full oral diet within 3 days. Benefit of immune-modulating formulae (enriched in arginin, nucleotides and $\dot{\omega}-3$ fatty acids) is proven in mild sepsis, reducing the number of bacteriemias and mortality but is unclear or even potentially harmful and, therefore, not recommended in severe sepsis (10).
On the other hand, EN is often associated with low amount of energy delivered. In such cases or when other obstacles occur adding parenteral nutrition (PN) to EN might be a tempting alternative but evidence is still insufficient for recommendation (16). It should be noted that EN is more frequently related to hypoglycemic events than total $\mathrm{PN}$ and mixed nutrition could be a way to reduce relevant hypoglycemias. $\mathrm{PN}$ is associated with higher risk of fungal and other superimposed bloodstream infections, too.

\section{CONCLUSION}

Although still controversial, intensive insulin therapy is the most widely used glycemic control method in ICU and, specifically, in septic patients. Negative impact of hyperglycemia is well-known and successfully avoided, but at the expense of unacceptable rates of hypoglycemic events despite recent advances in insulin administration and glucose monitoring. Because glycemic control is multifactorial, perhaps, the correct strategy is combined approach with computerized infusion, continuous BG monitoring and proper feeding support being the missing pieces in this sweet puzzle. Large statistically powered studies are necessary to provide the evidence-based medicine proof.

\section{REFERENCES}

1. Ali, N. A., J. M. O'Brien, Jr., K. Dungan, G. Phillips, C. B. Marsh, S. Lemeshow, et al. Glucose variability and mortality in patients with sepsis.Crit. Care Med., 36, 2008, No 8, 2316-2321.

2. Brunkhorst, F. M., C. Engel, F. Bloos, A. MeierHellmann, M. Ragaller, N. Weiler, et al. Intensive insulin therapy and pentastarch resuscitation in severe sepsis.- New Engl. J. Med., 358, 2008, No 2, 125-139.

3. Cappi, S. B., D. T. Noritomi, I. T. Velasco, R. Curi, T. C. Loureiro, F. G. Soriano. Dyslipidemia: a prospective controlled randomized trial of intensive glycemic control in sepsis.- Intensive Care Med., 38, 2012, No 4, 634-641.

4. Das, U. N. Insulin in sepsis and septic shock.- J. Assoc. Physicians India, 51, 2003, 695-700. 
5. Dellinger, R. P., M. M. Levy, J. M. Carlet, J. Bion, M. M. Parker, R. Jaeschke, et al.; Surviving Sepsis Campaign Guidelines Committee including The Pediatric Subgroup. Surviving Sepsis Campaign: international guidelines for management of severe sepsis and septic shock: 2008.- Crit. Care Med., 36, 2008, No 2, 296-327.

6. Egi, M., R. Bellomo, E. Stachowski, C. J. French, G. Hart. Variability of blood glucose concentration and short-term mortality in critically ill patients.Anesthesiology, 105, 2006, No 2, 244-252.

7. Finfer, S., D. R. Chittock, S. Y. Su, D. Blair, D. Foster, V. Dhingra, et al.; NICE-SUGAR Study Investigators. Intensive versus conventional glucose control in critically ill patients.- New Engl. J. Med., 360, 2009, No 13,1283-1297.

8. Garcia Branco R, Tasker RC, Ramos Garcia PC, Piva JP, Dias Xavier L. Glycemic control and insulin therapy in sepsis and critical illness.- J. Pediatr. (Rio J.), 83, 2007, Suppl. 5, S128-S136.

9. Griesdale, D. E., R. J. de Souza, R. M. van Dam, D. K. Heyland, D. J. Cook, A. Malhotra, et al. Intensive insulin therapy and mortality among critically ill patients: a meta-analysis including NICE-SUGAR study data.- CMAJ, 180, 2009, No 8, 821-827.

10. Kreymann, K. G., M. M. Berger, N. E. Deutz, M. Hiesmayr, P. Jolliet, G. Kazandjiev, et al.; DGEM; ESPEN. ESPEN Guidelines on Enteral Nutrition: Intensive care.- Clin. Nutr., 25, 2006, No 2, 210-223.

11. Langouche, L., S. Vander Perre, P. J. Wouters, A. D'Hoore, T. K. Hansen, G. Van den Berghe. Effect of intensive insulin therapy on insulin sensitivity in the critically ill.- J. Clin. Endocrinol. Metab., 92, 2007, No 10, 3890-3897.

12. Lorencio, C., Y. Leal, A. Bonet, J. Bondia, C. C. Palerm, A. Tache, et al. Real-time continuous glucose monitoring in an intensive care unit: better accuracy in patients with septic shock.- Diabetes Technol. Ther., 14, 2012, No 7, 568-575.
13. Preiser, J. C., P. Devos, S. Ruiz-Santana, C. Melot, D. Annane, J. Groeneveld, et al. A prospective randomised multi-centre controlled trial on tight glucose control by intensive insulin therapy in adult intensive care units: the Glucontrol study.Intensive Care Med., 35, 2009, No 10, 1738-1748.

14. Qaseem, A., L. L. Humphrey, R. Chou, V. Snow, P. Shekelle; Clinical Guidelines Committee of the American College of Physicians. Use of intensive insulin therapy for the management of glycemic control in hospitalized patients: a clinical practice guideline from the American College of Physicians.- Ann. Intern. Med., 154, 2011, No 4, 260-267.

15. Savioli, M., M. Cugno, F. Polli, P. Taccone, G. Bellani, P. Spanu, et al. Tight glycemic control may favor fibrinolysis in patients with sepsis.- Crit. Care Med., 37, 2009, No 2, 424-431.

16. Singer, P., M. M. Berger, G. Van den Berghe, G. Biolo, P. Calder, A. Forbes, et al.; ESPEN. ESPEN Guidelines on Parenteral Nutrition: intensive care.Clin. Nutr., 28, 2009, No 4, 387-400.

17. Szrama, J., P. Smuszkiewicz, I. Trojanowska. Glycemic profile and effectiveness and safety of insulin therapy in septic patients: is the blood glucose level sufficient?- Pol. Arch. Med. Wewn., 119, 2009, No 10, 621-627.

18. Van den Berghe, G. Insulin therapy in the intensive care unit should be targeted to maintain blood glucose between $4.4 \mathrm{mmol} / \mathrm{l}$ and $6.1 \mathrm{mmol} / \mathrm{l}$ Diabetologia, 51, 2008, No 6, 911-915.

19. van den Berghe, G., P. Wouters, F. Weekers, C. Verwaest, F. Bruyninckx, M. Schetz, et al. Intensive insulin therapy in critically ill patients.- New Engl. J. Med., 345, 2001, No 19, 1359-1367.

20. Waeschle, R. M., O. Moerer, R. Hilgers, P. Herrmann, P. Neumann, M. Quintel. The impact of the severity of sepsis on the risk of hypoglycaemia and glycaemic variability.- Crit. Care, 12, 2008, No 5, R129. doi: 10.1186/cc7097. 